\title{
Reviewer's comment concerning "The management of vertebral artery injury in anterior cervical spine operation: a systematic review of published cases" (DOI:10.1007/s00586-012-2423-8 by Hyung-Ki Park and Hae-Dong Jho)
}

\author{
Ronald H. M. A. Bartels
}

Received: 29 July 2012/Published online: 14 July 2012

(C) Springer-Verlag 2012

The authors have to be commended for their attempt to structure the various information reported in literature about this potentially devastating event during a very frequently performed surgical procedure for, in most instances, a benign entity. In their study, the use of a high-speed drill seemed to be related to a higher risk on lacerating the vertebral artery. It is not clear to me, if an aberrant course of the vertebral artery within vertebral body or in the proximity of the vertebral body was present in these cases. Every surgeon should carefully study the preoperative radiological examinations in order to identify a normal course of the vertebral artery. The first step to avoid damage to the vertebral artery is knowing its exact course and relation to the part of the spine of interest. The authors stated correctly that agreement does not exist due to the rarity of this event. By reviewing the literature, they tried to provide a treatment strategy. It should be emphasized that the review is not performed according to the MOOSE guidelines for reviewing observational studies and that the conclusions are based on studies of the lowest level of evidence. These considerations in combination with the need for a quick and adequate solution will contribute to the fact that a surgeon will not remember the proposed strategy at the moment of lacerating the vertebral artery. Dexterity, experience, and character of the surgeon will ultimately decide what solution is chosen. I believe that the recommendation of the authors for further studies with larger sample size is a standard phrase, and certainly will not be fulfilled.

Conflict of interest None.
R. H. M. A. Bartels ( $₫)$

Department of Neurosurgery, Radboud University Nijmegen

Medical Centre, R. Postlaan 4, 6500 HB Nijmegen,

The Netherlands

e-mail: R.Bartels@nch.umcn.nl 